\title{
DIFFUSION SYSTEMS REACTING AT THE BOUNDARY*
}

\author{
BY \\ W. E. OLMSTEAD \\ Northwestern University
}

\begin{abstract}
The problem considered is that of two species or chemical concentrations which independently diffuse within the same or adjacent regions. The coupling interaction takes place only along a common boundary. This boundary reaction is allowed to be either totally dissipative wherein both species are removed by the interaction, or semi-dissipative wherein one species is stimulated at the expense of the other. This physical situation is modeled by two independent, linear heat equations, each defined over a one-dimensional, semi-infinite domain. Associated with each heat equation is a boundary flux condition containing a nonlinear interactive term which couples the solutions of the two heat equations. With only boundary interaction, the problem can be reduced to the study of two coupled Volterra integral equations. By using monotone operator methods these integral equations are shown to have positive solutions. Uniqueness is also established. The large-time asymptotic behavior of the solutions is examined for the cases of both fast and slow decay of data.
\end{abstract}

1. Introduction. Let $u_{i}(x, t), i=1,2$, denote solutions to a system of two nonlinear boundary value problems involving the heat equation,

$$
\begin{aligned}
\gamma_{i} \frac{\partial^{2} u_{i}}{\partial x^{2}}(x, t) & =\frac{\partial u_{i}}{\partial t}(x, t), \quad x>0, \quad t>0, \\
\gamma_{i} \frac{\partial u_{i}}{\partial x}(0, t) & =\mu_{i} F\left[u_{1}(0, t), u_{2}(0, t)\right]-q_{i}(t), \quad t>0, \\
u_{i}(x, 0) & =U_{i}(x), \quad x \geq 0, \\
u_{i}(x, t) & \rightarrow 0 \quad \text { as } \quad x \rightarrow \infty, \quad t>0 .
\end{aligned}
$$

Here the $\gamma_{i}$ are given positive constants specifying the diffusivity associated with each solution. The $\mu_{i}= \pm 1$, with the choice depending upon the desired boundary effect. The $q_{i}(t)$ and $U_{i}(x)$ are given non-negative functions associated with the external input at the boundary and the initial data, respectively. The smooth convex function $F\left(u_{1}, u_{2}\right)$ provides the coupling of the system.

This mathematical problem has relevance to various physical situations. Perhaps the

\footnotetext{
* Received September 26, 1978. This work was supported by the National Science Foundation under Grant MCS 77-01327.
} 
simplest interpretation would be that of two chemicals coexisting and independently diffusing in some bath $x>0$. Their concentrations $u_{i}(x, t) \geq 0$ vary in space and time. At the surface $x=0$ of the bath, the chemicals react so as to influence the flux of concentration through the surface, and hence throughout the interior. In the case of an $m, n$ th-order reaction, it follows (cf. [1]) that

$$
F\left(u_{1}, u_{2}\right)=k_{m n} u_{1}^{m} u_{2}^{n}, \quad m, n=1,2, \ldots
$$

There has been considerable attention devoted to parabolic systems with nonlinear reaction effects (cf. [2] for references); however, these all have the reaction effects in the differential equation as opposed to the type of problem posed here. By considering (1.1)-(1.4) with coupling only on the boundary, it is possible to reduce the problem to that of two coupled Volterra integral equations for the surface concentrations, namely

$$
\phi(t) \equiv u_{1}(0, t), \quad \psi(t) \equiv u_{2}(0, t) .
$$

Moreover, since only the independent variable $t$ appears in the reduced form of the problem, this represents a considerably simpler setting for the investigation of complicated reaction effects.

To achieve the desired reduction of the problem, let

$$
x=\sqrt{ } \gamma_{1} \xi=\sqrt{ } \gamma_{2} \eta, \quad \alpha=1 / \sqrt{ } \gamma_{1}, \quad \beta=1 / \sqrt{ } \gamma_{2} .
$$

Then using the Green's function corresponding to the linear heat equation with zero flux condition at $x=0$, each of the boundary value problems (1.1)-(1.4) can be recast as an integral equation (cf. [3]). This yields

$$
\begin{aligned}
& \phi(t)=\int_{0}^{t}[\pi(t-s)]^{-1 / 2}\left\{f(s)-\alpha \mu_{1} F[\phi(s), \psi(s)]\right\} d s, \\
& \psi(t)=\int_{0}^{t}[\pi(t-s)]^{-1 / 2}\left\{h(s)-\beta \mu_{2} F[\phi(s), \psi(s)]\right\} d s, \quad t \geq 0,
\end{aligned}
$$

where

$$
\begin{aligned}
& f(t)=\alpha q_{1}(t)+\frac{1}{2 \pi^{1 / 2} t^{3 / 2}} \int_{0}^{\infty} U_{1}\left(\sqrt{ } \gamma_{1} \xi\right) \xi e^{-\xi^{2} / 4 t} d \xi, \\
& h(t)=\beta q_{2}(t)+\frac{1}{2 \pi^{1 / 2} t^{3 / 2}} \int_{0}^{\infty} U_{2}\left(\sqrt{ } \gamma_{2} \eta\right) \eta e^{-\eta^{2 / 4 t}} d \eta, \quad t \geq 0 .
\end{aligned}
$$

The purpose of this work will be to investigate the coupled nonlinear Volterra integral equations (1.8)-(1.9). By using monotone operator methods, the existence of positive solutions will be established. Uniqueness will be separately demonstrated. Finally, the asymptotic behavior as $t \rightarrow \infty$ will be examined for the special case of first-order reactions (i.e. $F(\phi, \psi) \equiv \phi \psi)$ when the data has algebraic decay.

In carrying out this investigation some distinction will be made between the totally dissipative reaction $\left(\mu_{1}, \mu_{2}\right)=(1,1)$, and the semi-dissipative reaction $\left(\mu_{1}, \mu_{2}\right)=(1,-1)$. The latter case is both more difficult technically and more interesting in terms of the physical interpretation of its behavior. 
2. Existence and uniqueness of positive solutions. To carry out the analysis which demonstrates the existence of positive solutions to (1.8)-(1.9), some reformulation is convenient. It is easily seen that this system is equivalent to

$$
\begin{aligned}
& \phi(t)=\omega \psi(t)+J(t), \quad J(t) \equiv \int_{0}^{t}[\pi(t-s)]^{-1 / 2}[f(s)-\omega h(s)] d s, \\
& \psi(t)=\int_{0}^{t}[\pi(t-s)]^{-1 / 2}\left\{h(s)-\frac{\alpha}{\omega} F[\omega \psi(s)+J(s), \psi(s)]\right\} d s,
\end{aligned}
$$

where

$$
\omega=\alpha \mu_{1} / \beta \mu_{2}=\alpha \mu_{2} / \beta \mu_{1}=\alpha / \beta \mu_{2}= \pm \alpha / \beta,
$$

with the plus sign corresponding to the totally dissipative case, while the minus sign designates the semi-dissipative case.

This form of the problem is conceptually more useful for the analysis, since the existence of $\psi(t)$ can be dealt with directly in (2.2), and then $\phi(t)$ follows from (2.1). The form of (2.2) is similar to the class of problems treated in [3]; however, the properties of the function $F(\phi, \psi)$ are more complicated here.

To produce positive solutions, it is appropriate to require that $J(t)$ be continuous and

$$
J(t) \geq 0, \quad t \geq 0 .
$$

For the reaction function $F(\phi, \psi)$, it is required that $F(\phi, \psi)$ be continuously differentiable in $\phi$ and $\psi$, with

$$
\begin{aligned}
F(\phi, \psi) & \geq 0, \quad F(0, \psi)=F(\phi, 0)=0, \quad \phi \geq 0, \quad \psi \geq 0, \\
\frac{\partial F}{\partial \phi}\left(\phi_{1}, \psi\right) & \geq \frac{\partial F}{\partial \phi}\left(\phi_{2}, \psi\right) \geq 0, \quad \phi_{1} \geq \phi_{2} \geq 0, \quad \psi \geq 0, \\
\frac{\partial F}{\partial \psi}\left(\phi, \psi_{1}\right) & \geq \frac{\partial F}{\partial \psi}\left(\phi, \psi_{2}\right) \geq 0, \quad \psi_{1} \geq \psi_{2} \geq 0, \quad \phi \geq 0 .
\end{aligned}
$$

Of course these properties are consistent with the special case (1.5) for the m,nth-order reaction.

The form of (2.2) is not appropriate for the application of monotone operator methods. To put it into the desired form, a more general Green's function $G_{\rho}\left(x, t \mid x^{\prime}, s\right)$ is used (cf. [3]) which satisfies the boundary condition

$$
\left.\frac{\partial G_{\rho}}{\partial x}\right|_{x=0}=\left.\rho(t) G_{\rho}\right|_{x=0}, \quad t>s .
$$

Here $\rho(t)$ is continuous and

$$
G_{\rho}(0, t \mid 0, s) \geq 0, \quad s<t<\infty, \text { when } \rho(t) \geq 0, \quad 0 \leq t<\infty .
$$

Furthermore,

$$
\int_{0}^{t} G_{\rho}(0, t \mid 0, s) \rho(s) d s<1, \quad 0 \leq t<\infty
$$


These properties are discussed in $[3,4]$, where it is also shown that an integral equation in the form of (2.2) can alternatively be considered in the form

$$
\psi(t)=T \psi(t) \equiv \int_{0}^{t} G_{\rho}(0, t \mid 0, s)\left|h(s)+\rho(s) \psi(s)-\frac{\alpha}{\omega} F[\omega \psi(s)+J(s), \psi(s)]\right| d s .
$$

It should be emphasized that subject to its continuity and positivity, $\rho(t)$ can be chosen as is convenient for the analysis. The choice $\rho(t) \equiv 0$ recovers $(2.2)$; however, the goal here will be to pick $\rho(t)$ so that $T$ becomes a monotone operator.

The existence results for (2.11) are obtained under somewhat different conditions for $\omega>0$ than for $\omega<0$. In light of the asymptotic results to follow in Sec. 3, the need for these different conditions is confirmed. In both cases, it will be shown that $T$ is a monotone operator on an appropriate space of non-negative functions. Let $P(t) \geq 0$ be a given continuous function and

$$
K_{P}=\left\{\psi(t): \psi(t) \in C_{0}[0, \infty), 0 \leq \psi(t) \leq P(t)\right\} .
$$

It will be seen that a different $P(t)$ is required for $\omega>0$ than for $\omega<0$. First consider $\omega>0$ and

THEOREM 1. Let $\omega>0$, and the hypotheses on $J(t)$ and $F(\phi, \psi)$ hold. Also let there exist a constant $M>0$ such that $0 \leq h(t) \leq(\alpha / \omega) F(\omega M, M)$. Then there exists a solution $\psi(t)$ of $(2.2)$ such that $\psi(t) \in K_{M}$.

Proof. Consider the alternative form (2.11) of (2.2) with the specification that

$$
\rho(t)=\frac{\alpha}{\omega} \frac{d F}{d \psi}(\omega M+J(t), M)
$$

where

$$
\frac{d F}{d \psi}=\omega \frac{\partial F}{\partial \phi}+\frac{\partial F}{\partial \psi} .
$$

To show that $T: K_{M} \rightarrow K_{M}$, suppose that $\psi \in K_{M}$; then $T \psi$ can be expressed as

$$
T \psi(t)=\int_{0}^{t} G_{\rho}\left|h+\left[\rho-\frac{\alpha}{\omega} \frac{d F}{d \psi}(\omega \hat{\psi}+J, \hat{\psi})\right] \psi\right| d s \geq 0
$$

for some $\hat{\psi} \in K_{M}$. The positivity of the integrand then follows from the definition of $\rho$ and the monotone properties of $\partial F / \partial \phi$ and $\partial F / \partial \psi$.

It is also possible to express $T \psi$ as

$$
\begin{aligned}
T \psi(t)= & \left.\int_{0}^{t} G_{\rho} \mid h-\frac{\alpha}{\omega} F(\omega M+J, M)+\left[\rho-\frac{\alpha}{\omega} \frac{d F}{d \psi}(\omega \psi+J, \psi)\right](\psi-M)\right\} d s \\
& +M \int_{0}^{t} \rho G_{\rho} d s \leq M \int_{0}^{t} \rho G_{\rho} d s \leq M,
\end{aligned}
$$

for some $\psi \in K_{M}$. The negativity of the first integral again follows from the definition of $\rho$ and the hypotheses of the theorem.

To show that $T$ is monotone on $K_{M}$, let $\psi_{1}, \psi_{2} \in K_{M}$ with $\psi_{1} \geq \psi_{2}$. Then $T \psi_{1}-T \psi_{2}$ can be expressed as

$$
T \psi_{1}-T \psi_{2}=\int_{0}^{t} G_{\rho}\left[\rho-\frac{\alpha}{\omega} \frac{d F}{d \psi}(\omega \bar{\psi}+J, \bar{\psi})\right]\left(\psi_{1}-\psi_{2}\right) d s \geq 0,
$$


for some $\bar{\psi} \in K_{M}$. The positivity of the integrand follows as before. Hence $T \psi_{1} \geq T \psi_{2}$ if $\psi_{1} \geq \psi_{2}$, thereby establishing the desired monotone property.

Finally, it is easily seen that

$$
0 \leq T 0 \leq T M \leq M .
$$

Thus the monotone operator theorem (cf. [5]) applies and provides that there exists a $\psi \in K_{M}$ such that $\psi=T \psi$. Moreover any solution of (2.11) is also a solution of (2.2).

Next consider $\omega<0$ and

Theorem 2. Let $\omega<0$, and the hypothesis on $J(t)$ and $F(\phi, \psi)$ hold. Let $N(t) \equiv$ $(1 /|\omega|) J(t)$. Then there exists a solution $\psi(t)$ of $(2.2)$ such that $\psi(t) \in K_{N}$.

Proof. Again consider the alternative form (2.11) of (2.2) with the specification that

$$
\rho(t)=\alpha \frac{\partial F}{\partial \psi}[J(t), J(t)] .
$$

To show that $T: K_{N} \rightarrow K_{N}$, suppose that $\psi \in K_{N}$; then $T \psi$ can be expressed as

$$
\left.T \psi=\int_{0}^{t} G_{\rho} \mid h+\left[\rho-\frac{\alpha}{\omega} \frac{d F}{d \psi}(\omega \hat{\psi}+J, \hat{\psi})\right] \psi\right\} d s \geq 0,
$$

for some $\hat{\psi} \in K_{N}$. The positivity of the integrand follows from the definition of $\rho$ and the monotone properties of $\partial F / \partial \phi$ and $\partial F / \partial \psi$ upon noting that

$$
\begin{aligned}
\rho-\frac{\alpha}{\omega} \frac{d F}{d \psi}(\omega \psi+J, \Psi) & =\rho-\alpha \frac{\partial F}{\partial \phi}(\omega \psi+J, \Psi)-\frac{\alpha}{\omega} \frac{\partial F}{\partial \psi}(\omega \psi+J, \Psi) \\
& \geq \rho-\alpha \frac{\partial F}{\partial \phi}(\omega \psi+J, \psi) \geq 0 .
\end{aligned}
$$

It is also possible to express $T \psi$ as

$$
\begin{aligned}
T \psi(t) & =\int_{0}^{t} G_{\rho}\left|\rho-\frac{\alpha}{\omega} \frac{d F}{d \psi}(\omega \psi+J, \psi)\right|(\psi-N) d s+\int_{0}^{t}(h+\rho N) G_{\rho} d s \\
& \leq \int_{0}^{t}(h+\rho N) G_{\rho} d s \leq N(t)
\end{aligned}
$$

for some $\psi \in K_{N}$. The negativity of the integrand of the first integral follows as in (2.20) and $\psi \leq N$. The final inequality in (2.21) follows from the same integral identity that permits (2.2) to be expressed as (2.11), namely

$$
\begin{aligned}
N(t) & =\frac{1}{|\omega|} J(t)=\int_{0}^{t}[\pi(t-s)]^{-1 / 2}\left[\frac{1}{|\omega|} f(s)+h(s)\right] d s \\
& =\int_{0}^{t} G_{\rho}\left[\frac{1}{|\omega|} f(s)+h(s)+\rho(s) N(s)\right] d s \\
& \geq \int_{0}^{t} G_{\rho}[h(s)+\rho(s) N(s)] d s .
\end{aligned}
$$


To show that $T$ is monotone on $K_{N}$, let $\psi_{1}, \psi_{2} \in K_{N}$ with $\psi_{1} \geq \psi_{2}$. Then $T \psi_{1}-T \psi_{2}$ can be expressed as

$$
T \psi_{1}-T \psi_{2}=\int_{0}^{t} G_{\rho}\left[\rho-\frac{\alpha}{\omega} \frac{d F}{d \psi}(\omega \bar{\psi}+J, \bar{\psi})\right]\left(\psi_{1}-\psi_{2}\right) d s \geq 0
$$

from some $\psi \in K_{N}$. The positivity of the integrand follows again as in (2.20). Hence $T \psi_{1} \geq T \psi_{2}$ if $\psi_{1} \geq \psi_{2}$, thereby establishing the desired monotone property.

Also, it follows that

$$
0 \leq T 0 \leq T N \leq N,
$$

and again the monotone operator theorem applies. Thus there exists a $\psi \in K_{N}$ such that $\psi=T \psi$, and that solution also satisfies (2.2).

Now that the existence of a $\psi(t) \geq 0$ which satisfies (2.2) has been demonstrated for both $\omega>0$ and $\omega<0$, it follows from $(2.1)$ that there exists a corresponding $\phi(t) \geq 0$. In the case $\omega>0$, it is readily apparent that $\phi(t) \geq 0$; whereas, for $\omega<0$ it should be noted that having shown $\psi(t) \leq(1 /|\omega|) J(t)$ is essential to insure that $\phi(t) \geq 0$.

To show uniqueness of the solutions established in Theorems 1 and 2, it suffices to prove that $(2.2)$ has only one continuous solution $\psi(t)$. The uniqueness of $\phi(t)$ then follows immediately from (2.1). The cases of $\omega>0$ and $\omega<0$ can be treated together in

TheOREM 3. There exists at most one $\psi(t) \in K_{P}$ which satisfies (2.2).

Proof. Suppose the contrary, namely that there exist $\psi_{1}, \psi_{2} \in K_{p}$ which satisfy (2.2). Let $\Psi \equiv \psi_{1}-\psi_{2}$, whereupon it is deduced from (2.2) that

$$
\Psi(t)=\int_{0}^{t}[\pi(t-s)]^{-1 / 2} v(s) \Psi(s) d s,
$$

where

$$
v(t)=-\frac{\alpha}{\omega} \frac{d F}{d \psi}[\omega \hat{\psi}(t)+J(t), \hat{\psi}(t)]
$$

for some $\hat{\psi} \in K_{p}$.

Since $\psi_{1}$ and $\psi_{2}$ are presumed continuous solutions of (2.2), then the properties of $F$ assure that $v$ is continuous. It is therefore possible to view (2.25) as a linear integral equation in $\Psi$ with a singular kernel $v(s)[\pi(t-s)]^{-1 / 2}$. Known uniqueness results on linear singular integral equations of this type (cf. [6]) provide that $\Psi \equiv 0$ is the only solution of (2.25). Thus, uniqueness for (2.2) is established.

3. Large-time behavior for first-order reactions. The investigation of the behavior of the solution $\psi(t)$ of $(2.2)$ as $t \rightarrow \infty$ is crucially dependent upon both the form of $F(\phi, \psi)$ and the sign of $\omega$. Here attention will be given only to the special case of first-order reactions where

$$
\alpha F(\phi, \psi) \equiv \phi \psi .
$$

A situation analogous to this with the nonlinearity appearing in the differential equations has been considered in [7].

For the special form of (3.1), both $\omega=1$ and $\omega=-1$ will be considered. The asymp- 
totic behavior will be examined when the data has algebraic decay. That is, it will be prescribed that as $t \rightarrow \infty$

$$
f(t) \sim A t^{-a}, \quad h(t) \sim B t^{-b} ; \quad A>0, \quad B>0, \quad 0 \leq a<b .
$$

Here the multiplicative constants $A$ and $B$ together with the decay rates $a$ and $b$ are taken as given. The condition $a<b$ is consistent with the specification that $J(t) \geq 0$. A distinction will be made between the situations of fast data decay $\left(a>\frac{3}{2}\right)$ and slow data decay $(b<1)$.

For the first-order reaction (3.1), the integral equation (2.2) takes the form

$$
\psi(t)=\int_{0}^{t}[\pi(t-s)]^{-1 / 2}\left|h(s)-\frac{1}{\omega} J(s) \psi(s)-\psi^{2}(s)\right| d s,
$$

where both the totally dissipative case $(\omega=1)$ and the semi-dissipative case $(\omega=-1)$ will be treated.

As found in [8,9], the asymptotic analysis of integral equations like (3.3) is often made easier by an Abel inversion of the integral operator to achieve the alternative form

$$
h(t)-\frac{1}{\omega} J(t) \psi(t)-\psi^{2}(t)=\frac{d}{d t} \int_{0}^{t}[\pi(t-s)]^{-1 / 2} \psi(s) d s .
$$

This form has the advantage that, in many instances, the right side is asymptotically unimportant to leading order. That is, the leading order balance is achieved between two of the three terms on the left side of (3.4).

For the asymptotic analysis here, it is assumed that as $t \rightarrow \infty$,

$$
\psi(t) \sim C t^{-c}, \quad C>0 .
$$

The requirement that $C$ is positive is consistent with the existence and uniqueness of a positive solution to (3.3) assured by Theorems $1-3$. While the solution does not necessarily have an asymptotic form like (3.5), it will be found to be self-consistent in the cases considered here. That is, $C$ and $c$ will be uniquely determined by a balance of leading order terms in (3.4) with other terms being of lower order.

A basic result needed in the asymptotic evaluation of the integral terms in (3.4) is that if a sufficiently smooth function $z(t)$ has asymptotic behavior as $t \rightarrow \infty$ of the form

$$
z(t) \sim D t^{-d},
$$

then, as $t \rightarrow \infty$,

$$
\begin{aligned}
\int_{0}^{t}[\pi(t-s)]^{-1 / 2} z(s) d s & \sim \frac{D \Gamma(1-d)}{\Gamma\left(\frac{3}{2}-d\right)} t^{1 / 2-d}, \quad d<1 \\
& \sim \frac{D}{\pi^{1 / 2}} t^{-1 / 2} \log t, \quad d=1 \\
& \sim \frac{\int_{0}^{\infty} z(s) d s}{\pi^{1 / 2}} t^{-1 / 2}, \quad d>1 .
\end{aligned}
$$

This follows from results given in [10]. 
Now the analysis of (3.4) can be done by utilizing the result (3.6)-(3.7). For fast data decay $\left(a>\frac{3}{2}\right)$, Eq. (3.4) has the asymptotic form

$$
\begin{aligned}
B t^{-b}-\frac{C}{\omega \pi^{1 / 2}} \int_{0}^{\infty}(f-\omega h) & d s t^{-c-1 / 2}-C^{2} t^{-2 c} \\
& =\frac{C \Gamma(1-c)\left(\frac{1}{2}-c\right)}{\Gamma\left(\frac{3}{2}-c\right)} t^{-c-1 / 2}, \quad c<1, \\
& =-\frac{C}{2 \pi^{1 / 2}} t^{-3 / 2} \log t, \quad c=1, \\
& =-\frac{\int_{0}^{\infty} \psi(s) d s}{2 \pi^{1 / 2}} t^{-3 / 2}, \quad c>1 .
\end{aligned}
$$

First examine the totally dissipative case $(\omega=1)$. By systematically considering every possible match of terms to leading order, it is determined that the only admissible result came from a balance of the first two terms on the left side (3.8), so that

$$
c=b-\frac{1}{2}, \quad C=B \pi^{1 / 2} / \int_{0}^{\infty}(f-h) d s .
$$

All other possible matchings are found to produce a contradiction, either in the sign of $C$, the lower bound on $a$, or an unbalanced dominant term. Thus follows that as $t \rightarrow \infty$

$$
\psi(t) \sim\left[B \pi^{1 / 2} / \int_{0}^{\infty}(f-h) d s\right] t^{1 / 2-b}, \quad \omega=1, \quad \frac{3}{2}<a<b .
$$

For the semi-dissipative case $(\omega=-1)$ a similar analysis reveals a balance to leading order of the latter two terms on the left side of (3.8). Again, all other possibilities lead to contradiction. Hence

$$
c=\frac{1}{2}, \quad C=\frac{1}{\pi^{1 / 2}} \int_{0}^{\infty}(f+h) d s,
$$

and it follows that

$$
\psi(t) \sim\left[\frac{1}{\pi^{1 / 2}} \int_{0}^{\infty}(f+h) d s\right] t^{-1 / 2}, \quad \omega=-1, \quad \frac{3}{2}<a<b .
$$

To examine the slow data decay $(b<1)$ situation, the asymptotic form of $(3.4)$ becomes

$$
\begin{aligned}
B t^{-b}-\frac{A C \Gamma(1-a)}{\omega \Gamma\left(\frac{3}{2}-a\right)} t^{1 / 2-a-c} & -C^{2} t^{-2 c} \\
& =\frac{C \Gamma(1-c)\left(\frac{1}{2}-c\right)}{\Gamma\left(\frac{3}{2}-c\right)} t^{-c-1 / 2}, \quad c<1, \\
& =-\frac{C}{2 \pi^{1 / 2} t^{-3 / 2} \log t, \quad c=1,} \\
& =-\frac{\int_{0}^{\infty} \psi(s) d s}{2 \pi^{1 / 2}} t^{-3 / 2}, \quad c>1 .
\end{aligned}
$$


Again examining the totally dissipative case $(\omega=1)$, it is found that the only possible matching is between the first two terms on the left side of (3.13), so that

$$
c=b-a+\frac{1}{2}, \quad C=\frac{B \Gamma\left(\frac{3}{2}-a\right)}{A \Gamma(1-a)} .
$$

Thus follows

$$
\psi(t) \sim \frac{B \Gamma\left(\frac{3}{2}-a\right)}{A \Gamma(1-a)} t^{-(b-a+1 / 2)}, \quad \omega=1, \quad 0 \leq a<b<1 .
$$

For the semi-dissipative case $(\omega=-1)$, the only possible matching is again between the latter two terms on the left side of (3.13), so that

$$
c=a-\frac{1}{2}, \quad C=\frac{A \Gamma(1-a)}{\Gamma\left(\frac{3}{2}-a\right)} .
$$

Thus follows that

$$
\psi(t) \sim \frac{A \Gamma(1-a)}{\Gamma\left(\frac{3}{2}-a\right)} t^{1 / 2-a}, \quad \omega=-1, \quad 0 \leq a<b<1 .
$$

The above results (3.10), (3.12), (3.15) and (3.17) cover each situation of fast data decay $\left(a>\frac{3}{2}\right)$ and slow data decay $(b<1)$ for either the totally dissipative effect $(\omega=1)$ or semi-dissipative effect $(\omega=-1)$. It is particularly noteworthy that, with either fast or slow data decay, the solution $\psi(t) \rightarrow 0$ as $t \rightarrow \infty$, except possibly in the case of $\omega=-1$, $0 \leq a \leq \frac{1}{2}$. For this situation of slow data decay in the semi-dissipative case, it is possible that $\psi(t)$ is non-decreasing as $t \rightarrow \infty$.

\section{REFERENCES}

[1] P. V. Danckwerts, Gas liquid reactions, McGraw-Hill, New York, 1970

[2] R. Aris, The mathematical theory of diffusion and reaction in permeable catalysts, Clarendon Press, Oxford, 1975

[3] W. E. Olmstead and R. A. Handelsman, Diffusion in a semi-infinite region with nonlinear surface dissipation, SIAM Review 18 (1976)

[4] W. E. Olmstead, A nonlinear integral equation associated with gas absorption in a liquid, Z. angew. Math. Phys. 28 (1977)

[5] M. A. Kransnosel'skii, Positive solutions of operator equations, P. Noordhoff, Groningen, 1964

[6] H. Hochstadt, Integral equations, Wiley, 1973

[7] C. S. Kahane, On a system of nonlinear parabolic equations arising in chemical engineering, J. Math. Anal. Appl. 53 (1976)

[8] R. A. Handelsman and W. E. Olmstead, Asymptotic solution to a class of nonlinear Volterra integral equations, SIAM J. Appl. Math. 22 (1972)

[9] W. E. Olmstead and R. A. Handelsman, Asymptotic solution to a class of nonlinear Volterra integral equations II, SIAM J. Appl. Math. 30 (1976)

[10] N. Bleistein and R. A. Handelsman, Asymptotic expansions of integrals, Holt, Rinehardt and Winston, New York (1975) 